\title{
Diagnosing Crohn's Disease: An economic analysis comparing wireless capsule endoscopy with traditional diagnostic procedures
}

\author{
Neil I. Goldfarb \\ Thomas Jefferson University \\ Laura T. Pizzi \\ Thomas Jefferson University \\ Joseph P. Fuhr Jr. \\ Thomas Jefferson University \\ Christopher Salvador

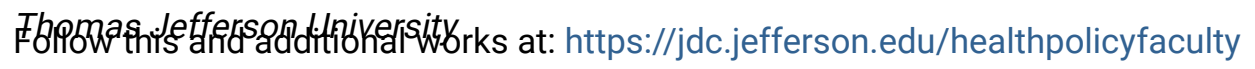 \\ nparabibfiltiea Health Services Research Commons \\ Therasisfeffersos Wpiversitw access to this document benefits you
}

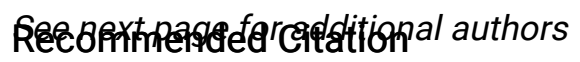

Goldfarb, Neil I.; Pizzi, Laura T.; Fuhr, Joseph P. Jr.; Salvador, Christopher; Sikirica, Vanja; Kornbluth, Asher; and Lewis, Blair, "Diagnosing Crohn's Disease: An economic analysis comparing wireless capsule endoscopy with traditional diagnostic procedures" (2004). College of Population Health Faculty Papers. Paper 11.

https://jdc.jefferson.edu/healthpolicyfaculty/11

This Article is brought to you for free and open access by the Jefferson Digital Commons. The Jefferson Digital Commons is a service of Thomas Jefferson University's Center for Teaching and Learning (CTL). The Commons is a showcase for Jefferson books and journals, peer-reviewed scholarly publications, unique historical collections from the University archives, and teaching tools. The Jefferson Digital Commons allows researchers and interested readers anywhere in the world to learn about and keep up to date with Jefferson scholarship. This article has been accepted for inclusion in College of Population Health Faculty Papers by an authorized administrator of the Jefferson Digital Commons. For more information, please contact: JeffersonDigitalCommons@jefferson.edu. 


\section{Authors}

Neil I. Goldfarb, Laura T. Pizzi, Joseph P. Fuhr Jr., Christopher Salvador, Vanja Sikirica, Asher Kornbluth, and Blair Lewis 


\title{
Diagnosing Crohn's Disease: An Economic Analysis Comparing Wireless Capsule Endoscopy with Traditional Diagnostic Procedures
}

\author{
NEIL I. GOLDFARB, B.A., LAURA T. PIZZI, Pharm.D., MPH, JOSEPH P. FUHR, JR., Ph.D., \\ CHRISTOPHER SALVADOR, Pharm.D., VANJA SIKIRICA, Pharm.D., \\ ASHER KORNBLUTH, M.D., and BLAIR LEWIS, M.D.
}

\begin{abstract}
The purpose of this study was to review economic considerations related to establishing a diagnosis of Crohn's disease, and to compare the costs of a diagnostic algorithm incorporating wireless capsule endoscopy (WCE) with the current algorithm for diagnosing Crohn's disease suspected in the small bowel. Published literature, clinical trial data on WCE in comparison to other diagnostic tools, and input from clinical experts were used as data sources for (1) identifying contributors to the costs of diagnosing Crohn's disease; (2) exploring where WCE should be placed within the diagnostic algorithm for Crohn's; and (3) constructing decision tree models with sensitivity analyses to explore costs (from a payor perspective) of diagnosing Crohn's disease using WCE compared to other diagnostic methods. Literature review confirms that Crohn's disease is a significant and growing public health concern from clinical, humanistic and economic perspectives, and results in a long-term burden for patients, their families, providers, insurers, and employers. Common diagnostic procedures include radiologic studies such as small bowel follow through (SBFT), enteroclysis, CT scans, ultrasounds, and MRIs, as well as serologic testing, and various forms of endoscopy. Diagnostic costs for Crohn's disease can be considerable, especially given the cycle of repeat testing due to the low diagnostic yield of certain procedures and the inability of current diagnostic procedures to image the entire small bowel. WCE has a higher average diagnostic yield than comparative procedures due to imaging clarity and the ability to visualize the entire small bowel. Literature review found the average diagnostic yield of SBFT and colonoscopy for work-up of Crohn's disease to be $53.87 \%$, whereas WCE had a diagnostic yield of $69.59 \%$. A simple decision tree model comparing two arms-colonoscopy and SBFT, or WCE-estimates that WCE produces a cost savings of \$291 for each case presenting for diagnostic work-up for Crohn's. Sensitivity analysis varying diagnostic yields of colonoscopy and SBFT vs. WCE demonstrates that WCE is still less costly than SBFT and colonoscopy even at their highest reported yields, as long as the diagnostic yield of WCE is $64.10 \%$ or better. Employing WCE as a first-line diagnostic procedure appears to be less costly, from a payor perspective, than current common procedures for diagnosing suspected Crohn's disease in the small bowel. Although not addressed in this model, earlier diagnosis with WCE (due to higher diagnostic yield) also could lead to earlier management, improved quality of life and workplace productivity for people with Crohn's disease. (Disease Management 2004;7:292-304)
\end{abstract}

Department of Health Policy, Jefferson Medical College, Philadelphia, Pennsylvania, and The Mount Sinai Medical Center, New York, New York. 


\section{INTRODUCTION}

C ROHN'S DISEASE (CD) is a type of inflammatory bowel disease which predominantly affects the small intestine and colon. ${ }^{1} \mathrm{CD}$ involves a transmural inflammation of the bowel wall and is characterized as discontinuous areas of inflammation, ulceration, scarring and fistualization throughout the GI tract, though primarily in the ileum, ileocecal region, and colon. ${ }^{2,3}$ Patients with CD can generally be categorized based on the location of the disease: small bowel alone $(30 \%-35 \%$ of cases), small and large bowel $(45 \%-50 \%$ of cases $)$ or colon alone (20\% of cases). ${ }^{2,4}$

Between 400,000 and 600,000 patients are believed to have CD in North America, with a reported incidence of 3.1 to 14.6 per 100,000 person-years in North America and a prevalence ranging from 26 to 198.5 per 100,000 people. ${ }^{5}$ In the United States, the incidence of CD is approximately 5 out of 100,000 and the prevalence is estimated to be 50 out of $100,000 .^{6}$ Although estimates of CD incidence vary across studies, all studies point to a substantial increase in cases of the disease over the past 30 years. ${ }^{1}$ Factors contributing to this increase are believed to include heightened provider and consumer awareness of Crohn's Disease, improved diagnostic testing, and changes in diet and behavioral risk factors. While CD affects both sexes, it may be more predominant in women, with the percentage of female cases ranging from $48 \%$ to $66 \% .^{5}$ Although CD can occur at any age, cases cluster within two distinct age ranges: $15-25$, and 50-80.1,7,8 The highest incidence of CD is seen in young adults, for whom chronic debilitating disease has broad societal implications ${ }^{9}$ both in terms of lifetime economic burden of disease, and lost productivity.

\section{Clinical presentation}

The clinical presentation of CD is often nonspecific but insidious, with symptoms fluctuating throughout the course of the disease. ${ }^{2}$ Symptoms may include diarrhea, abdominal pain, weight loss, fever, and blood in the stool. Clinical presentation is influenced by the pattern of injury, including the extent and severity of mucosal disease. ${ }^{9}$ Extraintestinal mani- festations of CD include rashes, arthritis, and uveitis as well as perirectal disease. ${ }^{2}$ Complications can include malnutrition, malabsorption, small bowel obstruction, anemia, malignancy of both the intestine and extra-intestinal sources, and bowel perforations. ${ }^{2,4}$ Less common, but serious complications include development of strictures of the intestinal tract leading to obstructive symptoms or fistulae.

\section{Treatment}

Treatment for $\mathrm{CD}$ can involve symptom management and/or control of disease activity. Medical therapies to limit symptoms include antidiarrheals, antispasmodics, and antibiotics. The inflammatory process can be modulated with the use of steroids (topical, enteral or parenteral), anti-inflammatory, antimetabolite and/or anti-TNF therapies. Disease remission also may be induced with surgical treatment. ${ }^{10,11}$ Although surgical resection of the involved intestinal segment is curative in some cases, recurrence of disease is common. Approximately $75 \%$ of patients with CD will need surgery at some point in their lives. In addition, $40 \%-50 \%$ of those with recurrent symptoms after a first operation will need a second operation, while $10 \%-30 \%$ will require a third operation. ${ }^{12}$ Although rarely fatal, CD is associated with high patient morbidity and, due to the early age of onset, can profoundly affect the patient's physical, social, professional and emotional well-being. ${ }^{13,14}$

\section{Economic impact}

In addition to its clinical consequences, CD carries a significant economic impact, ${ }^{15-17}$ with the total cost of illness estimated to exceed \$1.7 billion annually in the United States. ${ }^{18}$ These costs can be classified as direct, indirect, or intangible (Fig. 1). Direct costs are defined as expenses incurred due to the disease. Examples can include diagnostic work-up, medications, hospitalizations, physician time, surgery and complications associated with each. The estimated direct cost of CD in the United States during 1990 was $\$ 1.0-\$ 1.2$ billion, ${ }^{19}$ and the medical cost of managing CD has been estimated at \$9,197 (\$US 1996) per patient with CD annually. ${ }^{18}$ The initial diagnostic work-up rep- 


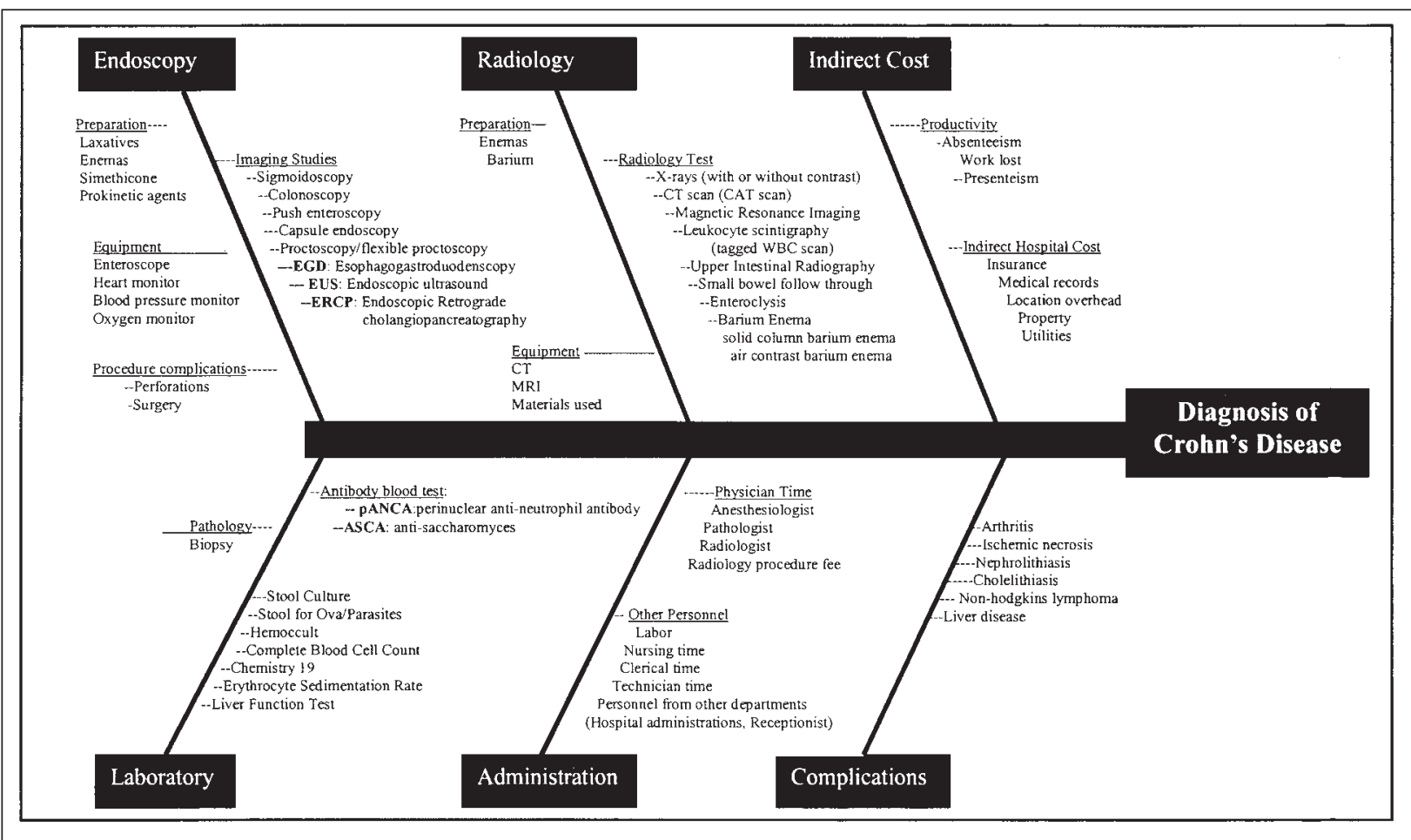

FIG. 1. Ishekawa diagram of costs associated with the diagnosis of Crohn's disease.

resents approximately $1.5 \%-7.8 \%$ of the total medical cost, and ranges between $\$ 140$ and \$650 (1996 US\$). ${ }^{15}$ Contributors to the direct cost for the initial diagnostic work-up include multiple endoscopic procedures, preparations for the procedures, equipment fees, imaging studies, radiology, laboratory charges, and personnel fees.

Indirect costs include lost productivity of patients and caregivers, transportation, and lodging expenses associated with receiving diagnostic testing and medical care. More severe cases of $C D$ could render the patient disabled and thus significantly impair productivity both at work and at home. Between $15 \%$ and $24 \%$ of patients are disabled after having CD for five or more years. ${ }^{18}$ The cost of CD-related disabilities has been estimated to be \$0.6-\$1.1 billion annually, with approximately $5 \%-10 \%$ of all patients with inflammatory bowel disease "so greatly incapacitated that they are unable to work."14,18

Intangible costs are associated with pain, anxiety, depression, altered body image, interference in interpersonal relations, sexual dysfunction and an overall decreased quality of life. ${ }^{18,20}$ Since CD is a chronic disease that typ- ically has an early age of onset, patients often suffer from the disease throughout most of their adult life and will need to develop coping skills in order to positively maintain their professional, social and personal lifestyles. ${ }^{18,20}$

\section{Establishing a diagnosis}

Procedures used to diagnose CD include imaging studies, radiologic tests, endoscopy, and serum antibody tests. ${ }^{3,21,22}$ Multiple tests and cycles of repeated testing are common in establishing a diagnosis. ${ }^{23}$ Because none of these tools represent a gold standard, the diagnosis is usually established through assessment of the clinical presentation in combination with radiographic, endoscopic and, if possible, histologic findings (Fig. 2) . 2,7,8,24 The National Cooperative Crohn's Disease Study reported an average lag time of 36 months between the onset of symptoms and the presence of abnormal findings on diagnostic evaluation. ${ }^{25}$

Initial diagnosis of $\mathrm{CD}$ has been difficult due to the limited diagnostic abilities of traditional technology. Despite the availability of a wide array of diagnostic tools, 2,3,21,22,26,27 early mucosal changes often cannot be detected using 


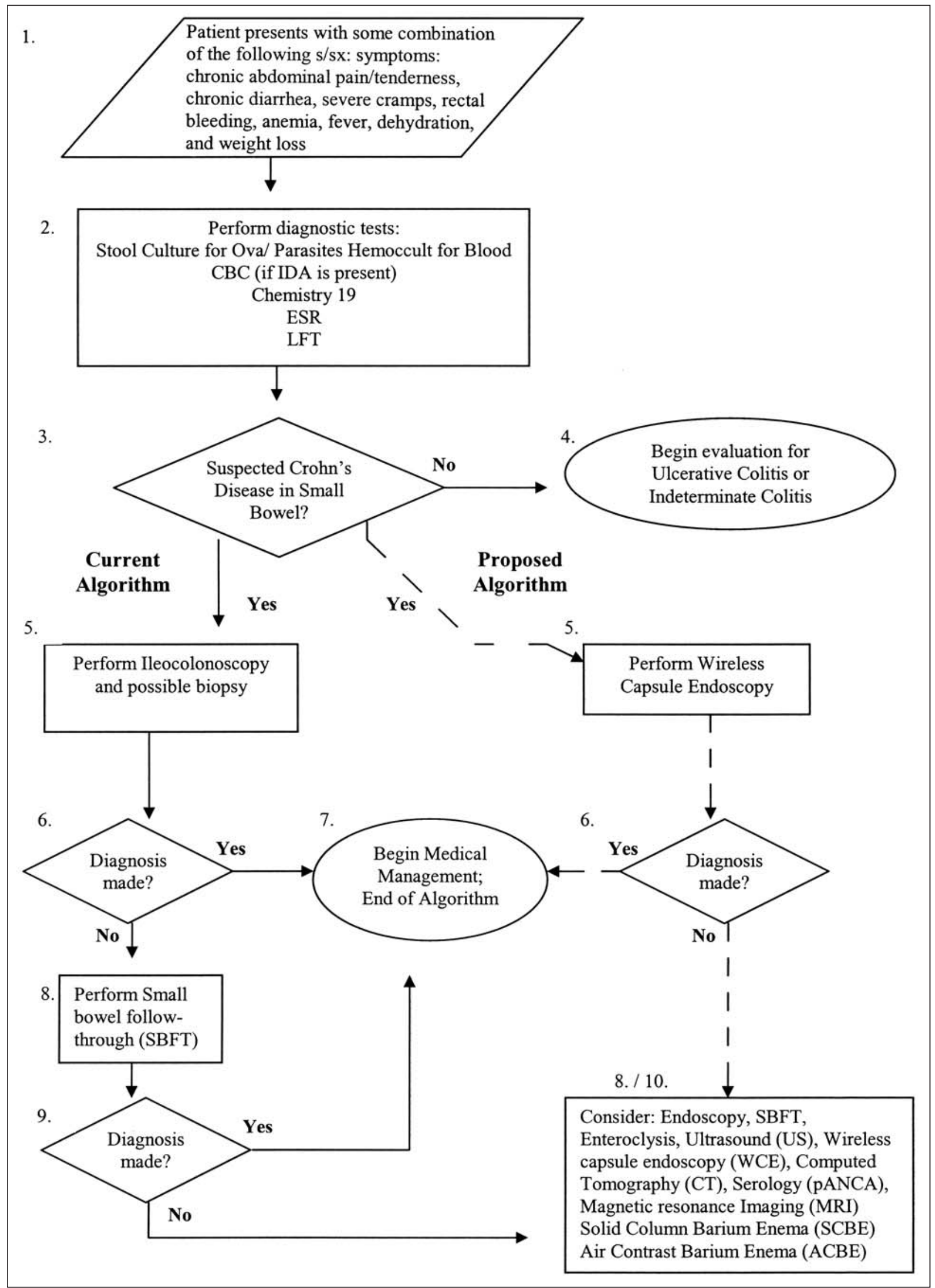

FIG. 2. Current and proposed algorithm for the diagnosis of Crohn's disease. 
traditional testing methods (particularly if these changes are located in the small bowel). Though $75 \%$ of patients with CD have small bowel involvement, more than one third have disease isolated to the small bowel. ${ }^{28}$

As a result of these diagnostic limitations, patients may be labeled as having an irritable bowel, and treatment with directed therapies that can control symptoms and place the CD in remission may not be initiated in a timely manner. (Conversely, patients with irritable bowel syndrome can be incorrectly labeled as having CD.) Since early intervention has a greater likelihood of inducing remission, clearly there is a strong need for a gold standard test to correctly diagnose $C D$ of the small bowel in its early stages.

Wireless capsule endoscopy (WCE) (Given Imaging Limited ${ }^{\mathrm{TM}}$, Yoqneam, Israel) received FDA clearance in 2001 for diagnosing diseases and disorders of the small intestine. The M2A ${ }^{\circledR}$ capsule, measuring $11 \times 26 \mathrm{~mm}$, obtains direct mucosal images from the entire small bowel. ${ }^{29}$ Images are transmitted by the capsule to a recording belt and are subsequently downloaded to a viewing station for review by a clinician. The early indications for WCE initially were to determine the source of occult or obscure gastrointestinal (GI) bleed, chronic GI blood loss or recurrent overt bleeding in patients with negative results of endoscopic examinations, and these indications were based upon initial published data. ${ }^{30}$ However, recent literature has shown that WCE provides improved imaging of the small bowel, $, 31,32$ and that WCE has higher diagnostic yields for CD in comparison to push enteroscopy, small bowel series ${ }^{31,33}$ and enteroclysis. ${ }^{34}$ With the high occurrence of CD in the small bowel and terminal ileum, WCE has emerged as a potential candidate for the initial diagnosis of CD. Based on a meta-analysis of 32 independent studies submitted to the FDA by Given Imaging, the FDA agreed to remove the "adjunctive tool" qualifier from the indications for WCE, making it available as a first-line tool in the imaging of the small bowel. ${ }^{32,35}$

In November 2003, Blue Cross and Blue Shield Association's Medical Advisory Panel determined that the use of capsule endoscopy for the initial diagnosis of patients with sus- pected CD meets TEC (Technology Evaluation Center) criteria. The TEC bases its assessments on clinical and scientific evidence and evaluates whether a technology improves health outcomes such as length of life, quality of life and ability to function.

The objective of the present study was to examine the clinical and economic factors associated with the current diagnosis of $C D$, and to compare the costs of a diagnostic algorithm employing WCE with the currently typical diagnostic algorithm using a decision tree model.

\section{MATERIALS AND METHODS}

A comprehensive evaluation of peer-reviewed published medical literature was conducted to review studies involving diagnostic procedures for CD. MEDLINE and CINAHL databases were used, with a search strategy that included the following keywords: "wireless capsule endoscopy," "video capsule endoscopy," "M2A capsule," "diagnosis," "differential diagnosis," "cost," and "Crohn's Disease." Separate searches were conducted on each of the potential diagnostic techniques that can be used in the diagnosis of CD: colonoscopy, endoscopy, magnetic resonance imaging (MRI), ultrasound (US), computed tomography (CT), small bowel follow through (SBFT), enteroclysis and serology tests. Because WCE was approved in August 2001, all searches on this device were limited to the English language with publication dates between January 2000 and February 2004. Longer search ranges were used for older diagnostic tools such as colonoscopy. References cited by the relevant articles were identified and reviewed (snowball technique). The search included studies of patients with suspected CD, and excluded studies involving patient populations with a history of previous inconclusive diagnostic work-up. Documents on the regulatory status of the WCE device, evidence-based guidelines from national professional organizations, and abstracts presented during Digestive Disease Week (DDW) 2003 were also reviewed.

A decision tree model was developed using TreeAge (Data Pro 4.0) software and Microsoft 
Excel spreadsheets. A payor perspective was taken, looking at direct medical costs for diagnostic testing. Cost data represented average Medicare reimbursements (technical and professional fees) for diagnostic procedures. ${ }^{36-38}$

\section{RESULTS}

\section{Review of diagnostic test options and yields}

1. Small bowel follow through (SBFT). Small bowel follow through (SBFT) is an established method for detecting and evaluating the presence of CD of the small bowel. Studies have shown its diagnostic yield in practice to be approximately $58 \%$ when CD is present. ${ }^{39,40}$ The primary advantage of SBFT is its ability to detect transmural thickening while being safer than colonoscopy. In addition, SBFT can be used in younger patients and requires less total time than enteroclysis. ${ }^{21,41}$ However, SBFT may miss changes of early Crohn's ileitis; it may be difficult to visualize and localize strictures, difficult to decipher whether lesions are raised or flat, and there is no ability to biopsy inherent to this procedure. ${ }^{21,39-41}$ Finally, with SBFT or any study involving the use of barium, there is the risk of radiation exposure and constipation. ${ }^{42}$

2. Enteroclysis. A number of studies suggest diagnostic superiority of enteroclysis, based on the ability of the evaluator to channel the contrast media into the bowel areas rather than having the patient ingest it as in SBFT. ${ }^{41,43} \mathrm{Al}-$ though enteroclysis may be more sensitive than SBFT, it has been reported to require a longer procedure time, involves more radiation exposure than routine small bowel series, ${ }^{44}$ and is more invasive since the physician must insert a tube into the patient's small intestine through the nose or mouth. ${ }^{21,34,45,46}$ Enteroclysis is commonly used in Europe. However, in the United States enteroclysis is not routinely available at many centers and is thus not widely used in the evaluation of Crohn's disease.

3. Colonoscopy. Colonoscopy is particularly useful in detecting colonic CD. It allows for visualization of the entire colon as well as the ter- minal ileum. In addition, the colonoscopy offers the inherent ability for conducting biopsies during the procedure. ${ }^{3}$ However, colonoscopy has several major disadvantages: (1) it is more invasive to the patient ${ }^{21,22,47} ;$ (2) it requires the use of intravenous conscious sedation which incurs extra costs and risks; and (3) the terminal ileum may be visualized only $70 \%-80 \%$ of the time, perhaps making it a suboptimal choice for detecting lesions in the small bowel, and endoscopic biopsies are not always possible. ${ }^{40,48-50}$ Given the latter, colonoscopy is generally not used alone in diagnosing CD; rather, it is typically conducted in combination with WCE, SBFT, or enteroclysis. ${ }^{51}$

\section{Single contrast barium enema (SCBE) and air} contrast barium enema ( $A C B E$ ). Barium enemas were once commonly used to evaluate patients with suspected colitis. Air contrast studies produce results with greater detail and are able to identify small ulcerations more frequently than single contrast studies. Barium enemas may also be used to evaluate the terminal ileum with reflux of barium into the ileum in retrograde fashion. Though this can be diagnostic for mucosal changes of CD, approximately $10 \%$ of studies will result in barium reflux into the small bowel, thus limiting the exam to the colon only. ${ }^{21}$

5. Computed tomography (CT). Computed tomography (CT) offers the ability to decipher the various types of patterns in which the disease may present ${ }^{21}$ and is useful for showing the site and cause of high-grade obstruction in patients. ${ }^{43}$ However, in order for CT to detect disease, tissue changes must extend the entire bowel wall, and include thickening. Solitary mucosal disease is not detected by this method. In addition, oral contrast given prior to the exam must reach the suspected area to provide an adequate exam. Its role in the initial diagnosis is limited due to the likelihood of false negatives resulting from an inability to detect early mucosal changes. More recently, the addition of multidetector machines to this exam has allowed the development of an improved technology called CT enterography. There are few published studies involving this technique, but preliminary data suggest improved detec- 
tion of bowel wall thickening and the presence of small bowel stenoses. ${ }^{21}$ Although this technique is improved for extra-luminal findings, it remains less sensitive than WCE for the detection of small bowel disease. ${ }^{33}$

6. Magnetic resonance imaging (MRI). Currently, magnetic resonance imaging (MRI) has a limited role in the evaluation of initial CD, in part due to limited accessibility and high costs. ${ }^{52}$ It is similar to CT in its cross-sectional imaging technique. It offers advantages over $\mathrm{CT}$, especially in evaluation of the perineal region, and may have superior tissue contrast, no radiation exposure, and ability to select various cross-sectional planes. ${ }^{53}$ Its main disadvantage in comparison to $\mathrm{CT}$ is poorer resolution.

7. Serology ( $p A N C A)$. Serology often is reserved as a supplemental tool to help rule-in or rule-out $\mathrm{CD}$ in cases which are questionable. There are two main tests that can be used; both rely on detecting serum antibodies. The perinuclear antineutrophil antibody (pANCA) and the anti-Saccharomyces cervisiae antibody (ASCA) are used to distinguish between Crohn's disease and ulcerative colitis (UC). Due to the low sensitivity of these tests, approximately $65 \%$ of patients with UC have pANCA in their blood, and approximately $40 \%$ of CD patients are ASCA positive. ${ }^{21,22}$ However, CD patients with only colonic involvement may be pANCA positive and ASCA negative. ${ }^{21}$

8. Wireless capsule endoscopy (WCE). Studies conducted to date have shown WCE to have an equivalent or higher diagnostic yield than other procedures, ${ }^{26,31,33,34,54-56}$ which suggests a reduced number of diagnostic procedures required to make an initial diagnosis of $C D$. The visual clarity is higher than that of barium studies. ${ }^{33}$ Like small bowel series or CT, WCE does not offer the ability to biopsy, but it can detect areas of abnormality for follow-up biopsy. WCE is more sensitive than other diagnostic procedures including barium studies and CT enterography in detecting abnormalities in the small bowel. ${ }^{31,32,35,55}$ In addition, it is less invasive than other procedures, thus promoting patients' compliance. A limitation of WCE is its potential for retention in the bowel due to strictures. The incidence of retention in work-up for suspected CD is unknown. However, retention occurred in $0.75 \%$ of cases from reported clinical trials, mostly performed for the evaluation of obscure bleeding. ${ }^{57}$ These cases of retention occurred secondary to small bowel tumors or strictures. The device is contraindicated in patients with known or suspected strictures, patients with pacemakers or other implanted electromedical devices, and patients with swallowing disorders. ${ }^{58}$ Patients with poor GI motility may not pass the capsule or the capsule might not visualize the entire small bowel. Patients with swallowing problems may not be able to swallow the capsule. ${ }^{30,59}$

\section{RESULTS OF THE DECISION TREE MODEL}

A decision tree model was constructed to compare typical current diagnostic work-up to work-up employing WCE as a first-line diagnostic test (Fig. 3). Typical work-up was defined as colonoscopy and SBFT based on consultation with clinician experts and literature review. Patients that fail to have a diagnosis made with these initial procedures (colonoscopy/SBFT) will then incur the cost of "other diagnostic tests." The model also takes into account the complications (ie, perforations) associated with colonoscopy/SBFT, as well as the associated cost of such events. The probability of perforation in patients undergoing colonoscopy was found to be $0.03 \%$ and the cost for treating colonoscopy-induced perforations was $\$ 20,000$ (US\$ 1990). ${ }^{60,61}$ In the comparative arm, patients would undergo WCE as the first-line procedure for the diagnosis. As with the current practice arm, patients that failed WCE would then incur the cost of "other diagnostic tests." This arm also takes into account the probability of capsule retention as well as the associated cost for overall retrieval. The probability of capsule retention was estimated to be $0.75 \%$ at most. ${ }^{62}$ The diagnostic failure rates for both procedures were based on the diagnostic yields found in the literature. The pooled yield of WCE, for all pathologies, across available studies, was found to be $69.59 \%$, while the pooled diagnostic yield of colonoscopy /SBFT was estimated to be $53.87 \%$ (Table 1). The cost of "other diagnostic tests" 


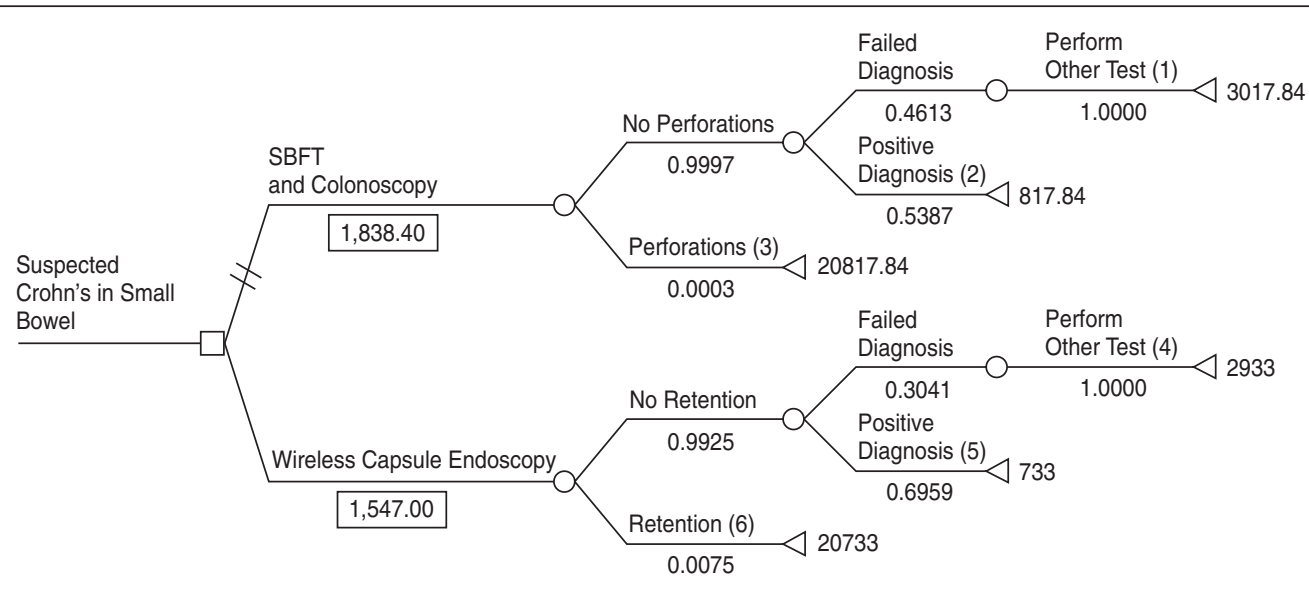

Estimated costs for "other tests," derived from the Hay and Hay study and adjusted to 2003 US dollars, was $\$ 2200$. Medicare costs for SFBT and Colonoscopy were $\$ 817.84$. Medicare costs for WCE were \&733.00. Costs for perforations and retention were estimated to be $\$ 20,000$.

${ }^{1}$ Total cost of other tests plus Medicare cost for SBFT and colonoscopy

${ }^{2}$ Total cost for positive test from SBFt and Colonoscopy

${ }^{3}$ Total cost for perforations and Medicare cost for SBFT and Colonoscopy

${ }^{4}$ Total cost of other tests plus Medicare cost for WCE

${ }^{5}$ Total cost for positive test from WCE

${ }^{6}$ Total cost for perforations and Medicare cost for WCE

FIG. 3. Economic analysis decision tree.

was derived from the diagnostic work-up in the Hay and Hay study. In order to employ more current figures, the numbers given in the Hay and Hay study (US\$1990) were converted to 2002 dollars by multiplying them by the annual increases in the medical care services component of the Consumer Price Index for the years 1990-2002. ${ }^{6}$

Physician and facility costs associated with each procedure were determined from reimbursement figures based upon Medicare Fee Schedule as determined by CMS. ${ }^{36-38}$ The total costs of both diagnostic arms (colonoscopy/SBFT versus WCE) were calculated and compared.

As shown in Figure 3, the total cost for patients undergoing colonoscopy/SBFT was calculated to be $\$ 1,838$ compared to $\$ 1,547$ for patients who underwent WCE. Thus, the net savings of using WCE, from a payor perspective, was estimated at \$291 per case initially presenting for work-up.

A sensitivity analysis was conducted to determine the robustness of our model to variation in assumptions in the range of diagnostic yields found in the published literature. Diagnostic yields of colonoscopy/SBFT and WCE were varied across the ranges as reported in the literature (Table 1). As long as the diagnostic yield for WCE was $64.10 \%$ or greater, WCE was the lower cost diagnostic option regardless of the diagnostic yield of colonoscopy/SBFT (across the range of yields reported in the literature, $45.24 \%-61.67 \%$ ). WCE also was the less costly first-line test, when comparing within the same diagnostic categories (ie, comparing high to high, average to average, or low to low).

In addition, a separate analysis was conducted comparing enteroclysis and colonoscopy versus WCE. The pooled diagnostic yield for enteroclysis and colonoscopy was found to be $40.04 \%$ with the cost of the combined procedure being $\$ 840$. All other figures in the model remained constant. The total cost for diagnosing Crohn's disease using enteroclysis and colonoscopy was $\$ 2,165$, compared to $\$ 1,547$ for WCE. Thus, the net savings of using WCE, from a payor perspective, was estimated at $\$ 618$ per case initially presenting for work-up. 


\begin{tabular}{|c|c|c|c|}
\hline & $\begin{array}{l}\text { Number } \\
\text { diagnosed }\end{array}$ & $\begin{array}{c}\text { Total number } \\
\text { evaluated }\end{array}$ & Diagnostic yield \\
\hline \multicolumn{4}{|l|}{ WCE } \\
\hline Fireman et al. 26 & 12 & 17 & $70.59 \%$ \\
\hline Chong et al. ${ }^{57}$ & 7 & 9 & $77.78 \%$ \\
\hline Eliakim et al. ${ }^{33}$ & 14 & 20 & $70.00 \%$ \\
\hline Costamagna et al. ${ }^{31 a}$ & 9 & 20 & $45.00 \%$ \\
\hline Mow et al. ${ }^{56 \mathrm{~b}}$ & 20 & 50 & $40.00 \%$ \\
\hline Hara et al. ${ }^{55}$ & 12 & 19 & $63.19 \%$ \\
\hline Buchmann et al. ${ }^{64}$ & 15 & 23 & $65.22 \%$ \\
\hline Bloom et al. 65 & 8 & 16 & $50.00 \%$ \\
\hline Tabibzadeh et al. ${ }^{66}$ & 35 & 44 & $79.55 \%$ \\
\hline Sum & 103 & 148 & $69.59 \%$ \\
\hline \multicolumn{4}{|l|}{ SBFT } \\
\hline Bernstein et al. ${ }^{40}$ & 15 & 26 & $57.69 \%$ \\
\hline Halligan et al. ${ }^{39}$ & 71 & 121 & $58.68 \%$ \\
\hline Buchmann et al. ${ }^{64}$ & 15 & 23 & $65.22 \%$ \\
\hline Bloom et al. ${ }^{65}$ & 9 & 16 & $56.25 \%$ \\
\hline Sum & 110 & 186 & $59.14 \%$ \\
\hline \multicolumn{4}{|l|}{ Enteroclysis } \\
\hline Bernstein et al. ${ }^{40}$ & 15 & 26 & $57.69 \%$ \\
\hline Cirillo et al. ${ }^{67}$ & 61 & 165 & $36.97 \%$ \\
\hline Maglinite et al. ${ }^{68}$ & 43 & 138 & $31.16 \%$ \\
\hline Sum & 119 & 329 & $36.17 \%$ \\
\hline \multicolumn{4}{|l|}{ Colonoscopy } \\
\hline Halligan et al. ${ }^{39}$ & 22 & 37 & $59.46 \%$ \\
\hline Coremans et al. ${ }^{46}$ & 48 & 110 & $43.64 \%$ \\
\hline Bloom et al. ${ }^{5}$ & 8 & 16 & $50.00 \%$ \\
\hline Sum & 78 & 163 & $47.85 \%$ \\
\hline
\end{tabular}

A sensitivity analysis was conducted for this model as well. Diagnostic threshold for WCE was determined while using the highest diagnostic yield for enteroclysis/colonoscopy $(58.73 \%)$. As long as the diagnostic yield for WCE was $60.13 \%$ or greater, WCE was the lower cost diagnostic option, regardless of the diagnostic yield of colonoscopy/enteroclysis (across the range of yields reported in the literature, $36.69 \%-58.73 \%$ ). As with the previous model, WCE was also the less costly first-line test when comparing within the same diagnostic categories (ie, comparing high to high, average to average, or low to low).

Certain assumptions were made in the creation of these models. Patients were assumed to be previously undiagnosed, present without strictures, and have disease detectable in the small bowel. ${ }^{2}$ The cost of additional tests used in the model was $\$ 2,200$ and was assumed to be the same in both arms. ${ }^{3}$ The cost of retrieval of capsule was assumed to be similar to the cost for treating colonoscopy-induced perforations through surgery $(\$ 20,000$ USD), when in fact capsule retrieval could be much lower due to spontaneous excretion or the ability to remove the capsule endoscopically. Therefore, this is a conservative estimate. It should be noted that surgical intervention for a retained capsule is likely to identify a diseased area (such as a tumor or stricture), while a repair for colonoscopy-induced perforation typically does not. The model does not address the added value of WCE for these rare events. ${ }^{4}$ Patients undergo both colonoscopy and SBFT in the control arm. Current clinical practice varies significantly, but in all likelihood literature indicates that, if anything, this model conservatively estimates costs of current (without WCE) standard diagnostic process. 


\section{DISCUSSION}

Literature review demonstrates that a number of tools are currently available for establishing a diagnosis of $\mathrm{CD}$. Our model illustrates that the higher diagnostic yield of WCE could translate into fewer diagnostic procedures for $\mathrm{CD}$, reducing total diagnostic costs.

This finding is further supported by the conservative diagnostic yields employed within the model. We used $69.59 \%$ and $53.87 \%$ for the diagnostic yields of WCE and colonoscopy/ SBFT, respectively. However, a meta-analysis of 32 independent studies submitted to the FDA by Given Imaging indicated a diagnostic yield of WCE of $71 \%$, with other diagnostic tests inclusive of SBFT, push-enteroscopy, CT scan, abdominal x-ray, angiography and interoperative enteroscopy having a pooled diagnostic yield of $41 \%$ across a variety of small bowel conditions. ${ }^{32,63}$

Our analysis focuses primarily on costs from a payor perspective. It therefore does not consider the additional potential benefits of earlier diagnosis associated with use of WCE, which remain to be studied. For example, since WCE is less invasive compared to colonoscopy/ SBFT and does not require the use of sedatives, anesthetics, or other medications, there is expected to be a psychological benefit which has not yet been quantified.

The societal implications of WCE include earlier diagnosis that can lead to earlier management and improved clinical outcomes. Through our literature review, we were able to estimate the number of diagnostic procedures conducted before $\mathrm{CD}$ diagnosis was made, and found this to be approximately 4.5 (Table 2). This finding is consistent with a long duration of symptoms prior to diagnosis. Earlier diagnosis holds the promise of earlier medical management and a reduced number of surgical interventions. Earlier management also is likely to improve patients' quality of life and reduce lost work productivity. These factors are expected to add to the overall cost savings of using WCE for diagnosing CD.

In addition, a negative capsule exam carries a diagnosis unlike a negative small bowel series. WCE has demonstrated a low miss rate of $0.5 \%$ for ulcers in the small bowel compared to

\begin{tabular}{|c|c|c|}
\hline \multicolumn{3}{|c|}{$\begin{array}{c}\text { Table 2. Average Number of Procedures } \\
\text { Per Patient Prior to WCE }\end{array}$} \\
\hline Study & $\begin{array}{l}\text { Number of } \\
\text { previous } \\
\text { procedures }\end{array}$ & $\begin{array}{l}\text { Number of } \\
\text { patients }\end{array}$ \\
\hline Costamagna et al..$^{31}$ & 210 & 22 \\
\hline Liangpunsakul et al. ${ }^{34}$ & 137 & 40 \\
\hline Eliakim et al. ${ }^{33}$ & 48 & 20 \\
\hline Herrerias et al. ${ }^{69}$ & 59 & 21 \\
\hline Fireman et al. ${ }^{26}$ & 92 & 17 \\
\hline Total & 546 & 120 \\
\hline $\begin{array}{l}\text { Average number } \\
\text { of procedures } \\
\text { per patient }\end{array}$ & 4.55 & \\
\hline
\end{tabular}

$78.7 \%$ for the comparison technologies. Therefore, a negative capsule exam is a true negative and small bowel Crohn's disease can effectively be dismissed from the differential diagnosis of a patient's symptoms. Therapy can then be directed toward another diagnosis such as irritable bowel syndrome.

The decision tree model presented in this analysis has several limitations. First, we did not employ a statistical meta-analysis for pooling the diagnostic yields available in the literature. Rather, we used an average of available yields, which was weighted based on the number of patients participating in each study. As such, our method fails to account for possible heterogeneity between the studies. However, given the limited number of studies assessing the yields of the included diagnostic alternatives, we decided to compute yields which were reflective of all available literature on the topic, rather than a statistically selected group of studies (which would have been the case had meta-analysis been used).

In addition, estimated diagnostic yields do not take into account the conjoint yields of tests used in combination. In pooling yields for SBFT and colonoscopy, the estimated combined yield is in fact lower than the yield of SBFT alone. However, the SBFT studies appear to significantly overestimate yields by including many patients with advanced disease. The model also uses a fairly conservative literature-based estimate of the costs of additional diagnostic testing associated with an undiagnosed case. It is quite possible that the lifetime (or longer-term) 
costs of diagnostic workup are significantly greater than what was used in the model, and any greater figure would only strengthen the case for WCE's early use in CD work-up. Although the model also does not incorporate the costs of confirmatory diagnostic testing (eg, biopsy to confirm a diagnosis by tissue histology), this testing is not clinically necessary if a diagnosis of CD has in fact been established.

Finally, our model assumes that colonoscopy is not conducted with WCE. Although many clinicians would argue that colonoscopy would be conducted as a matter of routine during the work-up process, we excluded it from the WCE arm because (1) studies have demonstrated that WCE alone has a sufficiently high yield such that other tests would not be required; (2) WCE is now accepted as a first-line method for imaging the small bowel; (3) the model is focused on initial diagnosis of $\mathrm{CD}$ for which there is expected to be minimal chance of retention (eg, due to minimal transmural thickening); and (4) the less invasive nature of WCE alone renders it more appropriate from the patient's perspective.

\section{CONCLUSION}

This economic analysis shows that employing WCE would most likely be less costly, from a payor perspective, than current diagnostic practice. WCE is an appropriate first-line test for diagnosing $C D$ and offers savings by reducing the number of tests required to establish a diagnosis. Through the earlier diagnosis made possible by WCE, patients are likely to receive appropriate treatment sooner than they would have under other diagnostic scenarios, and experience corresponding gains in quality of life.

\section{ACKNOWLEDGMENTS}

This study was supported by a grant from Given Imaging Inc., Yoqneam, Israel.

\section{REFERENCES}

1. Whelan G. Epidemiology of inflammatory bowel disease. Med Clin North Am 1990;74:1-12.
2. Fedorak R, Thomson A. Inflammatory Bowel Disease. In Thomson A and Schaffer E (ed). First Principles of Gastroenterology: The Basis of Disease and an Approach to Management. On-line version available at http:/ / gastroresource.com

3. Metcalf C. Crohn's disease: an overview. Nurs Standard 2002;16:45-52.

4. Sachar DB, Walfish JS. Crohn's Disease. In Merck Manual of Medical Information, Second Home Edition. Merck Research Laboratories, Whitehouse Station, NJ. 2003.

5. Loftus EV, Jr., Schoenfeld P, Sandborn WJ. The epidemiology and natural history of Crohn's disease in population-based patient cohorts from North America: a systematic review. Aliment Pharmacol Ther 2002;16:51-60.

6. Hanauer SB, Sandborn W. The practice parameters committee of the american college of gastroenterology. Management of Crohn's disease in adults. Am J Gastroenterol 2001;96:635-643.

7. Eisen GM, Sandler RM. Epidemiology and IBD. Crohn's and Colitis Foundation (CCFA). 2003.

8. MDConsult. Establishing the Diagnosis and Evaluation of Disease. 2003.

9. Cohen R. Inflammatory Bowel Disease Diagnosis and Therapeutics. Totowa, NJ: Humana Press, 2003.

10. Lombardi DA, Feller ER, Shah SA. Medical management of inflammatory bowel disease in the new millennium. Comprehens Ther 2002;28:39-49.

11. Feagan BG. Maintenance therapy for inflammatory bowel disease. Am J Gastroenterol 2003;98:S17.

12. Crohn's and Colitis Foundation (CCFA). Surgery for Crohn's disease [On-line]. Available: www.ccfa.org, 2003.

13. Cohen RD. The quality of life in patients with Crohn's disease. Aliment Pharmacol Ther 2002;16:1603-1609.

14. Feagan BG, Vreeland MG, Larson LR, et al. Annual cost of care for Crohn's disease: a payor perspective. Am J Gastroenterol 2000;95:1955-1960.

15. Hay AR, Hay JW. Inflammatory bowel disease: medical cost algorithms. J Clin Gastroenterol 1992;14: 318-327.

16. Hay JW, Hay AR. Inflammatory bowel disease: costsof-illness. J Clin Gastroenterol 1992;14:309-317.

17. Sen. H.M. Reid, Rep. S.W. Kelly. Inflammatory Bowel Disease Act HR 290/S491.

18. Hanauer SB, Cohen RD, Becker RV, III, et al. Advances in the management of Crohn's disease: economic and clinical potential of infliximab. Clin Ther 1998;20:1009-1028.

19. Feagan BG. Review article: economic issues in Crohn's disease-assessing the effects of new treatments on health-related quality of life. Aliment Pharmacol Ther 1999;13:S37.

20. Cohen RD. The quality of life in patients with Crohn's disease. Aliment Pharmacol Ther 2002;16:1603-1609.

21. American College of Radiology (ACR). American College of Radiology (ACR), expert Panel on Gastrointestinal Imaging. Imaging recommendations for patients with Crohn's disease, 2001. 
22. Crohn's and Colitis Foundation (CCFA). Introduction to diagnostic techniques [On-line]. Available: www.ccfa.org, 2003.

23. Goldfarb NI, Phillips A, Conn M, et al. Economic and health outcomes of capsule endoscopy: opportunities for improved management of the diagnostic process for obscure gastrointestinal bleeding. Dis Management 2002;5:123-135.

24. Kefalas C. Gastroduodenal Crohn's disease. Baylor University Medical Proc 2003;16:147-151.

25. Goldberg HI, Caruthers SB, Jr., Nelson JA, et al. Radiographic findings of the National Cooperative Crohn's Disease Study. Gastroenterology 1979;77:t-37.

26. Fireman Z, Mahajna E, Broide E, et al. Diagnosing small bowel Crohn's disease with wireless capsule endoscopy. Gut 2003;52:390-392.

27. Herrerias JM, Caunedo A, Rodriguez-Tellez M, et al. Capsule endoscopy in patients with suspected Crohn's disease and negative endoscopy. Endoscopy 2003;35:564-568.

28. Engstrom PF, Goosenberg EB. Diagnosis and Management of Bowel Diseases. Philadelphia, PA: Professional Communications, 1999.

29. Adler D, Gostout C. Wireless capsule endoscopy. Hosp Physician [On-line]. Available: www.turnerwhite.com/pdf/hp_may03_capsule.pdf, 2003.

30. Given ${ }^{\circledR}$ Diagnostic Imaging System-K010312 [Online]. Available: www.fda.gov, 2003.

31. Costamagna G, Shah SK, Riccioni ME, et al. A prospective trial comparing small bowel radiographs and video capsule endoscopy for suspected small bowel disease. Gastroenterology 2002;123:999-1005.

32. Meta Analysis Report: Performance Evaluation of Given Diagnostic System in the Diagnosis of Small Bowel Diseases and Disorders. Version 2, 1-36. 2003.

33. Eliakim R, Fischer D, Suissa A, et al. Wireless capsule video endoscopy is a superior diagnostic tool in comparison to barium follow-through and computerized tomography in patients with suspected Crohn's disease. Eur J Gastroenterol Hepatol 2003;15: 363-367.

34. Liangpunsakul S, Chadalawada V, Rex DK, Maglinte D, Lappas J. Wireless capsule endoscopy detects small bowel ulcers in patients with normal results from state of the art enteroclysis. Am J Gastroenterol 2003; 98:1295-1298.

35. Bowel Disorders Detection System Approved. FDAnews Daily Bull 2003;4[134].

36. Medicare Fees in the United States, PMIC. 2003.

37. Medicare RBRVS: The Physicians Guide. CMS Final Rule Published December 31, 2002.

38. Federal Register. 2003;68[60].

39. Halligan S, Saunders B, Williams C, Bartram C. Adult Crohn disease: can ileoscopy replace small bowel radiology? Abdom Imaging 1998;23:117-121.

40. Bernstein CN, Boult IF, Greenberg HM, van der PW, Duffy G, Grahame GR. A prospective randomized comparison between small bowel enteroclysis and small bowel follow-through in Crohn's disease. Gastroenterology 1997;113:390-398.
41. Gurian L, Jendrzejewski J, Katon R, Bilbao M, Cope R, Melnyk C. Small-bowel enema. An underutilized method of small-bowel examination. Dig Dis Sci 1982;27:1101-1108.

42. Kelvin FM, Maglinte DD. Enteroclysis or small bowel follow-through in Crohn' s diseases? Gastroenterology 1998;114:1349-1351.

43. Nolan DJ. The true yield of the small-intestinal barium study. Endoscopy 1997;29:447-453.

44. Ott DJ, Chen YM, Gelfand DW, Van Swearingen F, Munitz HA. Detailed per-oral small bowel examination vs. enteroclysis. Part II: Radiographic accuracy. Radiology 1985;155:31-34.

45. Maglinte DD, Chernish SM, Kelvin FM, O'Connor KW, Hage JP. Crohn disease of the small intestine: accuracy and relevance of enteroclysis. Radiology 1992;184:541-545.

46. Coremans G, Rutgeerts P, Geboes K, Van den OJ, Ponette E, Vantrappen G. The value of ileoscopy with biopsy in the diagnosis of intestinal Crohn's disease. Gastrointest Endosc 1984;30:167-172.

47. Zwas FR, Bonheim NA, Berken CA, Gray S. Ileoscopy as an important tool for the diagnosis of Crohn's disease: a report of seven cases. Gastrointest Endosc 1994;40:89-91.

48. Geboes K, Vantrappen G. The value of colonoscopy in the diagnosis of Crohn's disease. Gastrointest Endosc 1975;22:18-23.

49. Geboes K. The value of endoscopic biopsies in the diagnosis of Crohn's disease. Tijdschrift Gastro-Enterologie 1978;21:87-97.

50. Holdstock G, DuBoulay CE, Smith CL. Survey of the use of colonoscopy in inflammatory bowel disease. Dig Dis Sci 1984;29:731-734.

51. Lewis BS. Ileoscopy should be part of standard colonoscopy: a comparison of radiographic and endoscopic evaluation of the ileum. J Clin Gastroenterol 2000;31:103-104.

52. Ebinger M, Rieber A, Leidl R. Cost-effectiveness of magnetic resonance imaging and enteroclysis in the diagnostic imaging of Crohn's disease. Int J Technol Assess Health Care 2002;18:711-717.

53. Rieber A, Wruk D, Potthast S, et al. Diagnostic imaging in Crohn's disease: comparison of magnetic resonance imaging and conventional imaging methods. Int J Colorectal Dis 2000;15:176-181.

54. Herrerias JM, Caunedo A, Rodriguez-Tellez M, Pellicer F, Herrerias JM, Jr. Capsule endoscopy in patients with suspected Crohn's disease and negative endoscopy. Endoscopy 2003;35:564-568.

55. Hara AK, Leighton JA, Sharma VK, Fleischer DE. Small bowel: preliminary comparison of capsule endoscopy with barium study and CT. Radiology 2004;230:260-265.

56. Mow W, Simon K, Targan S, Dubinsky M, Treyzon L, Abreu-Martin $\mathrm{M}$ et al. Initial experience with wireless capsule enteroscopy in the diagnosis and management of inflammatory bowel disease. Clin Gastroenterol Hepatol 2004;2:31-40.

57. Chong AK, Taylor AC, Miller AM, Desmond PV. Ini- 
tial experience with capsule endoscopy at a major referral hospital. Med J Aust 2003;178:537-540.

58. Given ${ }^{\circledR}$ Diagnostic Imaging System-K010312 [Online]. Available: www.fda.gov, 2003.

59. Yu M. M2A capsule endoscopy. A breakthrough diagnostic tool for small intestine imaging. Gastroenterol Nurs 2002;25:24-27.

60. Korman LY, Overholt BF, Box T, Winker CK. Perforation during colonoscopy in endoscopic ambulatory surgical centers. Gastrointest Endosc 2003;58:554-557.

61. Wagner JL, Herdman RC, Wadhwa S. Cost effectiveness of colorectal cancer screening in the elderly. Ann Intern Med 1991;115:807-817.

62. Barkin J. Wireless capsule endoscopy. Med Clin North Am 2004;14:51-65.

63. FDA Establishes Capsule Endoscopy as First-Line Test for Imaging Small Bowel Mucosa. Clinical Practice and Economics. AGA Policy Update. 7-24-2003.

64. Buchmann A, Miller F, Wallin A. Videocapsule endoscopy: a blinded comparison to small bowel contrast study in the diagnosis of Crohn's disease. Dig Dis Week 2003;S1355.

65. Bloom P, Rosenberg M, Klein S, et al. Wireless capsule endoscopy (CE) is more informative than ileoscopy and SBFT for the evaluation of the small intestine (SI) in patients with known or suspected Crohn's disease. Dig Dis Week 2003;S1367.
66. Tabibzadeh S, Kimble J, Zaidel O, et al. Utility of wireless capsule enteroscopy (WCE) versus serology in the evaluation of small bowel Crohn's disease. Dig Dis Week 2003;M1889.

67. Cirillo LC, Camera L, Della NM, Castiglione F, Mazzacca G, Salvatore M. Accuracy of enteroclysis in Crohn's disease of the small bowel: a retrospective study. Eur Radiol 2000;10:1894-1898.

68. Maglinte DD, Chernish SM, Kelvin FM, O'Connor KW, Hage JP. Crohn disease of the small intestine: accuracy and relevance of enteroclysis. Radiology 1992;184:541-545.

69. Herrerias JM, Caunedo A, Rodriguez-Tellez M, Pellicer F, Herrerias JM, Jr. Capsule endoscopy in patients with suspected Crohn's disease and negative endoscopy. Endoscopy 2003;35:564-568.

Address reprint requests to: Dr. Neil Goldfarb

Department of Health Policy Jefferson Medical College

1015 Walnut St., Ste. 115 Philadelphia, PA 19107

E-mail: neil.goldfarb@jefferson.edu 